\title{
Set Integral Equations in Metric Spaces
}

\author{
IOANA TişE
}

Abstract. Let $P_{c p, c v}\left(\mathbb{R}^{n}\right)$ be the family of all nonempty compact, convex subsets of $\mathbb{R}^{n}$.

We consider the following set integral equations:

$$
\begin{aligned}
& X(t)=\int_{a}^{b} K(t, s, X(s)) \mathrm{d} s+X_{0} \\
& X(t)=\int_{a}^{t} K(t, s, X(s)) \mathrm{d} s+X_{0},
\end{aligned}
$$

where $K:[a, b] \times[a, b] \times P_{c p, c v}\left(\mathbb{R}^{n}\right) \rightarrow P_{c p, c v}\left(\mathbb{R}^{n}\right)$ and $X_{0} \in P_{c p, c v}\left(\mathbb{R}^{n}\right)$.

The purpose of the paper is to study the existence and data dependence of the solutions of the set integral equations (1) and (2), by using a fixed point approach. Our results generalize and extend the results given in [2]. For other similar results see [3] and [4].

\section{INTRODUCTION}

Let $\mathbb{R}^{n}$ be the real $n$-dimensional Euclidian space and $P_{c p, c v}\left(\mathbb{R}^{n}\right)$ be the family of all nonempty compact, convex subset of $\mathbb{R}^{n}$ endowed with the Pompeiu-Hausdorff metric $H$. It is well-known that $\left(P_{c p, c v}\left(\mathbb{R}^{n}\right), H\right)$ is a complete metric space.

We consider the following set integral equations:

$$
\begin{aligned}
& X(t)=\int_{a}^{b} K(t, s, X(s)) \mathrm{d} s+X_{0}, \\
& X(t)=\int_{a}^{t} K(t, s, X(s)) \mathrm{d} s+X_{0},
\end{aligned}
$$

where $K:[a, b] \times[a, b] \times P_{c p, c v}\left(\mathbb{R}^{n}\right) \rightarrow P_{c p, c v}\left(\mathbb{R}^{n}\right)$.

A solution of (1) or (2) means a continuous function $X:[a, b] \rightarrow P_{c p, c v}\left(\mathbb{R}^{n}\right)$ which satisfies (1) respectively (2) for each $t \in[a, b]$.

The purpose of the article is to study the existence and data dependence of the solutions of the equation (1) and (2). The approach is based on the well-known Banach-Caccioppoli contraction principle. Our results generalize

2000 Mathematics Subject Classification. Primary: 47H10.

Key words and phrases. Fixed point, set integral equation, Pompeiu-Hausdorff metric. 
and extend the results given in [2]. For other similar results see [3], [5] and [4].

The paper is organized as follows. Next section, Preliminaries, contains some basic notations and notions used through the paper. Third section presents existence and the data dependence results of the solution for the equations (1) and (2).

\section{Preliminaries}

The aim of this section is to present some notions and symbols used in the paper.

Let us define the following generalized functionals:

$$
D: P\left(\mathbb{R}^{n}\right) \times P\left(\mathbb{R}^{n}\right) \rightarrow \mathbb{R}_{+}, \quad D(A, B)=\inf \{d(a, b) \mid a \in A, b \in B\} .
$$

$D$ is called the gap functional between $A$ and $B$. In particular, if $x_{0} \in X$ then $D\left(x_{0}, B\right):=D\left(\left\{x_{0}\right\}, B\right)$.

$$
\rho: P\left(\mathbb{R}^{n}\right) \times P\left(\mathbb{R}^{n}\right) \rightarrow \mathbb{R}_{+} \cup\{+\infty\}, \quad \rho(A, B)=\sup \{D(a, B) \mid a \in A\} .
$$

$\rho$ is called the (generalized) excess functional.

$$
H: P\left(\mathbb{R}^{n}\right) \times P\left(\mathbb{R}^{n}\right) \rightarrow \mathbb{R}_{+} \cup\{+\infty\}, H(A, B)=\max \{\rho(A, B), \rho(B, A)\} .
$$

$H$ is the (generalized) Pompeiu-Hausdorff functional.

It is known that $\left(P_{c p, c v}\left(\mathbb{R}^{n}\right), H\right)$ is a complete metric space ([1]).

Lemma 2.1 ([6]). Let $X$ be a Banach space. Then

$$
H(A+C, B+D) \leq H(A, B)+H(C, D) \text {, for } A, B, C, D \in P(X) .
$$

Proof. Let $\varepsilon>0$. From the definition of $H$ it follows that there exists $(a+c) \in A+C$ such that $D(a+c, B+D) \geq H(A+C, B+D)-\varepsilon$ or exists $(b+d) \in B+D$ such that $D(b+d, A+C) \geq H(A+C, B+D)-\varepsilon$.

Let us consider the first case. For $a, c$ we get $b \in B, d \in D$ such that:

$$
\|a-b\| \leq H(A, B)+\frac{\varepsilon}{2}, \quad\|c-d\| \leq H(C, D)+\frac{\varepsilon}{2} .
$$

Then $H(A+C, B+D)-\varepsilon \leq D(a+c, B+D) \leq\|(a+c)-(b+d)\|$ we obtain that $H(A+C, B+D)-\varepsilon \leq H(A, B)+H(C, D)+\varepsilon$, proving the desired inequality.

\section{MAin RESUlts}

We consider on $C\left([a, b], P_{c p, c v}\left(\mathbb{R}^{n}\right)\right)$ the metric:

$$
H_{*}(X, Y):=\max _{t \in[a, b]} H(X(t), Y(t)) .
$$

The pair $\left(C\left([a, b], P_{c p, c v}\left(\mathbb{R}^{n}\right)\right), H_{*}\right)$ forms a complete metric space.

Our first result is an existence and uniqueness theorem for the solution of the equation (1). 
Theorem 3.1. Let $K:[a, b] \times[a, b] \times P_{c p, c v}\left(\mathbb{R}^{n}\right) \rightarrow P_{c p, c v}\left(\mathbb{R}^{n}\right)$ be a multivalued operator. Suppose that:

(i) $K$ is continuous on $[a, b] \times[a, b] \times P_{c p, c v}\left(\mathbb{R}^{n}\right)$ and $X_{0} \in P_{c p, c v}\left(\mathbb{R}^{n}\right)$,

(ii) $K(t, s, \cdot)$ is Lipschitz, i.e., there exists $L_{K} \geq 0$ such that:

$$
H(K(t, s, A), K(t, s, B)) \leq L_{K} H(A, B),
$$

for all $A, B \in P_{c p, c v}\left(\mathbb{R}^{n}\right)$ and for all $t, s \in[a, b]$,

(iii) $L_{K}(b-a)<1$.

Then the set integral equation

$$
X(t)=\int_{a}^{b} K(t, s, X(s)) \mathrm{d} s+X_{0}
$$

has a unique solution.

Proof. Consider the operator: $\Gamma: P_{c p, c v}\left(\mathbb{R}^{n}\right) \rightarrow P_{c p, c v}\left(\mathbb{R}^{n}\right)$ defined for each $t \in[a, b]$ by

$$
\Gamma X(t)=\int_{a}^{b} K(t, s, X(s)) \mathrm{d} s+X_{0} .
$$

We need to verify the contraction condition for $\Gamma$.

$$
\begin{aligned}
& H(\Gamma(X)(t), \Gamma(Y)(t))= \\
= & H\left(\int_{a}^{b} K(t, s, X(s)) \mathrm{d} s+X_{0}, \int_{a}^{b} K(t, s, Y(s)) \mathrm{d} s+X_{0}\right) \\
\leq & H\left(X_{0}, X_{0}\right)+H\left(\int_{a}^{b} K(t, s, X(s)) \mathrm{d} s, \int_{a}^{b} K(t, s, Y(s)) \mathrm{d} s\right) \\
\leq & \int_{a}^{b} H(K(t, s, X(s)), K(t, s, Y(s))) \mathrm{d} s \\
\leq & \int_{a}^{b} L_{K} H(X(s), Y(s)) \mathrm{d} s .
\end{aligned}
$$

Taking the maximum for $t \in[a, b]$, then we have:

$$
\begin{aligned}
\max _{t \in[a, b]} H(\Gamma(X)(t), \Gamma(Y)(t)) & \leq L_{K} \int_{a}^{b} \max _{t \in[a, b]} H(X(s), Y(s)) \mathrm{d} s \\
H_{*}(\Gamma(X), \Gamma(Y)) & \leq L_{K}(b-a) H_{*}(X, Y),
\end{aligned}
$$

for all $t \in[a, b]$, and $X, Y \in C\left([a, b], P_{c p, c v}\left(\mathbb{R}^{n}\right)\right)$.

Thus, the integral operator $\Gamma$ is Lipschitz with constant $L_{\Gamma}=L_{K}(b-a)<$ 1. From the contraction principle we get the result.

A data dependence result for the solution of equation (1) is: 
Theorem 3.2. Let $K_{1}, K_{2}:[a, b] \times[a, b] \times P_{c p, c v}\left(\mathbb{R}^{n}\right) \rightarrow P_{c p, c v}\left(\mathbb{R}^{n}\right)$, be continuous. Consider the following set equations:

$$
\begin{aligned}
& X(t)=\int_{a}^{b} K_{1}(t, s, X(s)) \mathrm{d} s+X_{0} \\
& Y(t)=\int_{a}^{b} K_{2}(t, s, Y(s)) \mathrm{d} s+Y_{0}
\end{aligned}
$$

Suppose:

(i) there exists $L_{K} \geq 0$ such that $H(K(t, s, A), K(t, s, B)) \leq L_{K} H(A, B)$, for all $A, B \in P_{c p, c v}\left(\mathbb{R}^{n}\right)$ and $t, s \in[a, b]$, with $L_{K}(b-a)<1$ (denote by $X^{*}$ the unique solution of the equation (3));

(ii) there exists $\eta_{1}, \eta_{2}>0$ such that:

(a) $H\left(K_{1}(t, s, U), K_{2}(t, s, U)\right) \leq \eta_{1}$, for all $(t, s, U) \in[a, b] \times[a, b] \times$ $P_{c p, c v}\left(\mathbb{R}^{n}\right)$, and

(b) $H\left(X_{0}, Y_{0}\right) \leq \eta_{2}$;

(iii) there exists $Y^{*}$ a solution of the equation (4).

Then

$$
H_{*}\left(X^{*}, Y^{*}\right) \leq \frac{\eta_{2}+\eta_{1}(b-a)}{1-L_{K}(b-a)}
$$

Proof. We have:

$$
\begin{aligned}
& H\left(X^{*}(t), Y^{*}(t)\right)= \\
= & H\left(\int_{a}^{b} K_{1}\left(t, s, X^{*}(s)\right) \mathrm{d} s+X_{0}, \int_{a}^{b} K_{2}\left(t, s, Y^{*}(s)\right) \mathrm{d} s+Y_{0}\right) \\
\leq & H\left(\int_{a}^{b} K_{1}\left(t, s, X^{*}(s)\right) \mathrm{d} s, \int_{a}^{b} K_{2}\left(t, s, Y^{*}(s)\right) \mathrm{d} s\right)+H\left(X_{0}, Y_{0}\right) \\
\leq & H\left(\int_{a}^{b} K_{1}\left(t, s, X^{*}(s)\right) \mathrm{d} s, \int_{a}^{b} K_{1}\left(t, s, Y^{*}(s)\right) \mathrm{d} s\right)+ \\
& +H\left(\int_{a}^{b} K_{1}\left(t, s, Y^{*}(s)\right) \mathrm{d} s, \int_{a}^{b} K_{2}\left(t, s, Y^{*}(s)\right) \mathrm{d} s\right)+\eta_{2} \\
\leq & \int_{a}^{b} H\left(K_{1}\left(t, s, X^{*}(s)\right), K_{1}\left(t, s, Y^{*}(s)\right)\right) \mathrm{d} s+ \\
& \quad+\int_{a}^{b} H\left(K_{1}\left(t, s, Y^{*}(s)\right), K_{2}\left(t, s, Y^{*}(s)\right)\right) \mathrm{d} s+\eta_{2} \\
\leq & \int_{a}^{b} H\left(K_{1}\left(t, s, X^{*}(s)\right), K_{1}\left(t, s, Y^{*}(s)\right)\right) \mathrm{d} s+\int_{a}^{b} \eta_{1} \mathrm{~d} s+\eta_{2} .
\end{aligned}
$$


By taking the maximum for $t \in[a, b]$, then we have:

$$
\begin{gathered}
\max _{t \in[a, b]} H\left(X^{*}(t), Y^{*}(t)\right) \leq \\
\max _{t \in[a, b]}\left(L_{K}(b-a) H\left(X^{*}(t), Y^{*}(t)\right)+\eta_{1}(b-a)+\eta_{2}\right) \\
\leq L_{K}(b-a) \max _{t \in[a, b]} H\left(X^{*}(t), Y^{*}(t)\right)+\eta_{1}(b-a)+\eta_{2} \\
\max _{t \in[a, b]} H\left(X^{*}(t), Y^{*}(t)\right) \leq \frac{\eta_{2}+\eta_{1}(b-a)}{1-L_{K}(b-a)} \\
H_{*}\left(X^{*}, Y^{*}\right) \leq \frac{\eta_{2}+\eta_{1}(b-a)}{1-L_{K}(b-a)} .
\end{gathered}
$$

We will prove now an existence result and a data dependence result for the solution of the equation (2).

We consider on $C\left([a, b], P_{c p, c v}\left(\mathbb{R}^{n}\right)\right)$ the metric:

$$
H_{*}^{B}(X, Y):=\max _{t \in[a, b]}\left[H(X(t), Y(t)) e^{-\tau(t-a)}\right] \text {, with arbitrary } \tau>0 .
$$

The pair $\left(C\left([a, b], P_{c p, c v}\left(\mathbb{R}^{n}\right)\right), H_{*}^{B}\right)$ forms a complete metric space.

Theorem 3.3. Let $K:[a, b] \times[a, b] \times P_{c p, c v}\left(\mathbb{R}^{n}\right) \rightarrow P_{c p, c v}\left(\mathbb{R}^{n}\right)$ be an operator. Suppose that:

(i) $K$ is continuous on $[a, b] \times[a, b] \times P_{c p, c v}\left(\mathbb{R}^{n}\right)$ and $X_{0} \in P_{c p, c v}\left(\mathbb{R}^{n}\right)$,

(ii) $K(t, s, \cdot)$ is Lipschitz, i.e., there exists $L_{K} \geq 0$ such that

$$
H(K(t, s, A), K(t, s, B)) \leq L_{K} H(A, B),
$$

for all $A, B \in P_{c p, c v}\left(\mathbb{R}^{n}\right)$ and $t, s \in[a, b]$.

Then the set integral equation

$$
X(t)=\int_{a}^{t} K(t, s, X(s)) \mathrm{d} s+X_{0}
$$

has a unique solution.

Proof. Consider the operator $\Gamma: P_{c p, c v}\left(\mathbb{R}^{n}\right) \rightarrow P_{c p, c v}\left(\mathbb{R}^{n}\right)$ defined for each $t \in[a, b]$ by

$$
\Gamma X(t)=\int_{a}^{t} K(t, s, X(s)) \mathrm{d} s+X_{0} .
$$


We will prove the contraction condition for $\Gamma$.

$$
\begin{aligned}
& H(\Gamma(X)(t), \Gamma(Y)(t))= \\
= & H\left(\int_{a}^{t} K(t, s, X(s)) \mathrm{d} s+X_{0}, \int_{a}^{t} K(t, s, Y(s)) \mathrm{d} s+X_{0}\right) \leq \\
\leq & H\left(X_{0}, X_{0}\right)+H\left(\int_{a}^{t} K(t, s, X(s)) \mathrm{d} s, \int_{a}^{t} K(t, s, Y(s)) \mathrm{d} s\right) \leq \\
\leq & \int_{a}^{t} H(K(t, s, X(s)), K(t, s, Y(s))) \mathrm{d} s \leq \int_{a}^{t} L_{K} H(X(s), Y(s)) \mathrm{d} s= \\
= & L_{K} \int_{a}^{t} H(X(s), Y(s)) e^{-\tau(s-a)} e^{\tau(s-a)} \mathrm{d} s \leq \\
\leq & L_{K} H_{*}^{B}(X, Y) \int_{a}^{t} e^{\tau(s-a)} \mathrm{d} s= \\
= & \frac{L_{K}}{\tau} H_{*}^{B}(X, Y)\left(e^{\tau(t-a)}-1\right) \leq \frac{L_{K}}{\tau} H_{*}^{B}(X, Y) e^{\tau(t-a)} .
\end{aligned}
$$

Then we have:

$$
\begin{gathered}
H(\Gamma(X)(t), \Gamma(Y)(t)) e^{-\tau(t-a)} \leq \frac{L_{K}}{\tau} H_{*}^{B}(X, Y) \\
H_{*}^{B}(\Gamma(X), \Gamma(Y)) \leq \frac{L_{K}}{\tau} H_{*}^{B}(X, Y),
\end{gathered}
$$

for all $t \in[a, b], X, Y \in C\left([a, b], P_{c p, c v}\left(\mathbb{R}^{n}\right)\right), \tau>0$.

Hence, we can apply the Banach contraction principle for $\Gamma$, since by choosing $\tau>L_{K}$, we get $L_{\Gamma}:=\frac{L_{K}}{\tau}<1$. By the contraction principle, the proof is complete.

Remark 3.1. Theorem 3.3 in this paper is a special case of Hammerstein's equality. General solution of this equality is given in [7].

A data dependence result is:

Theorem 3.4. Let $K_{1}, K_{2}:[a, b] \times[a, b] \times P_{c p, c v}\left(\mathbb{R}^{n}\right) \rightarrow P_{c p, c v}\left(\mathbb{R}^{n}\right)$ be continuous. Consider the following set equations:

$$
\begin{aligned}
& X(t)=\int_{a}^{t} K_{1}(t, s, X(s)) \mathrm{d} s+X_{0} \\
& Y(t)=\int_{a}^{t} K_{2}(t, s, Y(s)) \mathrm{d} s+Y_{0}
\end{aligned}
$$

Suppose:

(i) $H(K(t, s, A), K(t, s, B)) \leq L_{K} H(A, B)$, for all $A, B \in P_{c p, c v}\left(\mathbb{R}^{n}\right)$ and $t, s \in[a, b]$, where $L_{K} \geq 0$ (denote by $X^{*}$ the unique solution of the equation (5));

(ii) there exists $\eta_{1}, \eta_{2}>0$, such that $H\left(K_{1}(t, s, U), K_{2}(t, s, U)\right) \leq \eta_{1}$, for all $(t, s, U) \in[a, b] \times[a, b] \times P_{c p, c v}\left(\mathbb{R}^{n}\right)$ and $H\left(X_{0}, Y_{0}\right) \leq \eta_{2}$; 
(iii) There exists $Y^{*}$ a solution of the equation (6).

Then

$$
H_{*}^{B}\left(X^{*}, Y^{*}\right) \leq \frac{\eta_{2}+\eta_{1}(b-a)}{1-\frac{L_{K}}{\tau}} e^{-\tau(b-a)}\left(\text { where } \tau>L_{K}\right) .
$$

Proof. We have:

$$
\begin{aligned}
& H\left(X^{*}(t), Y^{*}(t)\right)= \\
= & H\left(\int_{a}^{t} K_{1}\left(t, s, X^{*}(s)\right) \mathrm{d} s+X_{0}, \int_{a}^{t} K_{2}\left(t, s, Y^{*}(s)\right) \mathrm{d} s+Y_{0}\right) \\
\leq & H\left(\int_{a}^{t} K_{1}\left(t, s, X^{*}(s)\right) \mathrm{d} s, \int_{a}^{t} K_{2}\left(t, s, Y^{*}(s)\right) \mathrm{d} s\right)+H\left(X_{0}, Y_{0}\right) \\
\leq & H\left(\int_{a}^{t} K_{1}\left(t, s, X^{*}(s)\right) \mathrm{d} s, \int_{a}^{t} K_{1}\left(t, s, Y^{*}(s)\right) \mathrm{d} s\right)+ \\
& \quad+H\left(\int_{a}^{t} K_{1}\left(t, s, Y^{*}(s)\right) \mathrm{d} s, \int_{a}^{t} K_{2}\left(t, s, Y^{*}(s)\right) \mathrm{d} s\right)+\eta_{2} \\
\leq & \int_{a}^{t} H\left(K_{1}\left(t, s, X^{*}(s)\right), K_{1}\left(t, s, Y^{*}(s)\right)\right) \mathrm{d} s+ \\
& \quad+\int_{a}^{t} H\left(K_{1}\left(t, s, Y^{*}(s)\right), K_{2}\left(t, s, Y^{*}(s)\right)\right) \mathrm{d} s+\eta_{2} \\
\leq & \int_{a}^{t} H\left(K_{1}\left(t, s, X^{*}(s)\right), K_{1}\left(t, s, Y^{*}(s)\right)\right) \mathrm{d} s+\int_{a}^{t} \eta_{1} \mathrm{~d} s+\eta_{2} \\
\leq & \int_{a}^{t} L_{K} H\left(X^{*}(s), Y^{*}(s)\right) e^{-\tau(s-a)} e^{\tau(s-a)} \mathrm{d} s+\int_{a}^{t} \eta_{1} \mathrm{~d} s+\eta_{2} .
\end{aligned}
$$

By taking the maximum for $t \in[a, b]$, we have:

$$
\begin{aligned}
& \max _{t \in[a, b]}\left(H\left(X^{*}(t), Y^{*}(t)\right) e^{-\tau(t-a)} e^{\tau(t-a)}\right) \leq \\
\leq & \max _{t \in[a, b]}\left(\int_{a}^{t} L_{K} H\left(X^{*}(s), Y^{*}(s)\right) e^{-\tau(s-a)} e^{\tau(s-a)} d s+\int_{a}^{t} \eta_{1} \mathrm{~d} s+\eta_{2}\right), \\
& H_{*}^{B}\left(X^{*}, Y^{*}\right) e^{\tau(b-a)}=L_{K} H_{*}^{B}\left(X^{*}, Y^{*}\right) \int_{a}^{t} e^{\tau(s-a)} \mathrm{d} s+\eta_{1}(b-a)+\eta_{2}= \\
& \frac{L_{K}}{\tau} H_{*}^{B}\left(X^{*}, Y^{*}\right)\left(e^{\tau(t-a)}-1\right)+\eta_{1}(b-a)+\eta_{2} \\
\leq & \frac{L_{K}}{\tau} H_{*}^{B}\left(X^{*}, Y^{*}\right) e^{\tau(b-a)}+\eta_{1}(b-a)+\eta_{2}, \\
& H_{*}^{B}\left(X^{*}, Y^{*}\right) \leq \frac{\eta_{2}+\eta_{1}(b-a)}{1-\frac{L_{K}}{\tau}} e^{-\tau(b-a)} .
\end{aligned}
$$


For $\tau>L_{K}$, we have

$$
H_{*}^{B}\left(X^{*}, Y^{*}\right) \leq \frac{\eta_{2}+\eta_{1}(b-a)}{1-\frac{L_{K}}{\tau}} e^{-\tau(b-a)} .
$$

\section{REFERENCES}

[1] J.P. Aubin, H. Frankowska, Set-Valued Analysis, Birkhauser, Basel, 1990.

[2] A.J. Brandao, F.S. De Blasi, F. Iervillino, Uniqueness and Existence Theorems for Differential Equations with Compact Convex Valued Solutions, Boll. Unione Mat. Ital., IV. Ser. 3(1970), 47-54.

[3] F.S. de Blasi, Semifixed sets of maps in hyperspaces with application to set differential equations, Set-Valued Anal., 14(2006), No. 3, 263-272.

[4] G.N. Galanis, T.G. Bhaskar, V. Lakshmikantham and P.K. Palamides, Set valued functions in Fréchet spaces: continuity, Hukuhara differentiability and applications to set differential equations, Nonlinear Anal., 61 (2005), No. 4, 559-575.

[5] V. Lakshmikantham, T. Gnana Bhaskar and J. Vasundhara Devi, Theory of Set Differential Equations in Metric Spaces, Cambridge Scientific Publishers, 2006.

[6] A. Petruşel, G. Petruşel, G. Mot, Topics in Nonlinear Analysis and Applications to Mathematical Economics, House of the Book Science, Cluj-Napoca, 2007.

[7] M.R. Taskovic, A Characterization of the Class of Contraction Type Mappings, Kobe Journal of Mathematics, 2 (1985), 45-55.

Department of Applied Mathematics, BABEş-Bolyai University

KogăLniCEANU 1, 400084

Cluj-NAPOCA,

ROMANIA

E-mail address: ti_camelia@yahoo.com 